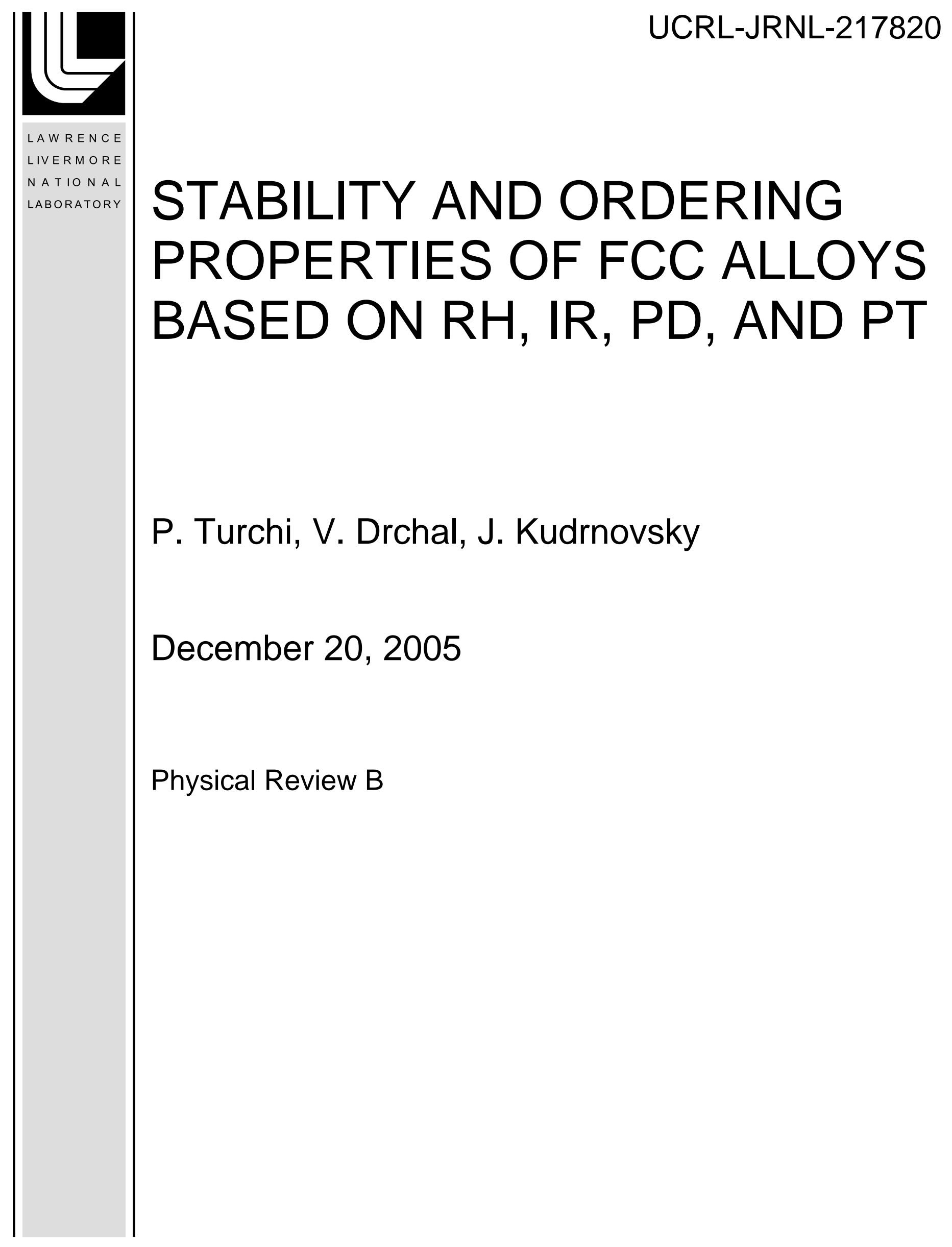


This document was prepared as an account of work sponsored by an agency of the United States Government. Neither the United States Government nor the University of California nor any of their employees, makes any warranty, express or implied, or assumes any legal liability or responsibility for the accuracy, completeness, or usefulness of any information, apparatus, product, or process disclosed, or represents that its use would not infringe privately owned rights. Reference herein to any specific commercial product, process, or service by trade name, trademark, manufacturer, or otherwise, does not necessarily constitute or imply its endorsement, recommendation, or favoring by the United States Government or the University of California. The views and opinions of authors expressed herein do not necessarily state or reflect those of the United States Government or the University of California, and shall not be used for advertising or product endorsement purposes. 


\title{
Stability and ordering properties of fcc alloys based on Rh, Ir, $\mathrm{Pd}$, and Pt
}

\author{
P. E. A. Turchi \\ Lawrence Livermore National Laboratory (L-372), P.O. Box 808, Livermore, CA 94551, USA
}

\author{
V. Drchal and J. Kudrnovský \\ Institute of Physics, Academy of Sciences of the Czech Republic, Na Slovance 2, CZ-182 21 Praha \\ 8, Czech Republic
}

(August 29, 2006)

\begin{abstract}
Stability properties and ordering trends for the six face-centered cubic binary combinations of the four transition metals $\mathrm{Rh}, \mathrm{Ir}, \mathrm{Pd}$, and Pt are examined in the context of electronic structure calculations. The method is based on a Green function description of the electronic structure of random alloys. Configurational order is treated within the generalized perturbation method. On one hand, the three alloys, Pd-Rh, Pd-Ir and Pt-Ir that have been studied experimentally are confirmed to behave like phase separating systems. On the other hand, the other three mixtures, Pd-Pt, Rh-Ir, and Pt-Rh for which phase separating trends have been inferred from experiments are found to display chemical order with ordering of the $\left(\begin{array}{lll}1 & 0 & 0\end{array}\right),\left(\begin{array}{lll}1 & 1 / 2 & 0\end{array}\right)$ family-types and a mixture of both, respectively. The origin of these results is discussed in term of electronic structure properties.

61.66.Dk, 64.60.Cn, 65.40.Ba, 71.20.Be
\end{abstract}

Typeset using REVTEX 


\section{INTRODUCTION}

The six phase diagrams associated with the possible combinations of two elements among the four fcc-based transition metals of columns VIIIA, Rh, Ir, Pd, and Pt as presented in Ref. [1] display deceptively simple features. Indeed, for each of the six alloy systems, the phase diagram indicates a miscibility gap at relatively low temperature in the solid phase, and a region of face-centered cubic (fcc) solid solution at high temperature in the entire range of composition before melting occurs. In only three among the six cases, namely for Pd-Rh, Pd-Ir, and Pt-Ir, the miscibility gap is represented by a solid line, indicating, by convention, that only in these cases the assessments have been based on actual experiments. Interestingly enough, all these diagrams have been proposed by Raub in the late fifties [2] in a review study on platinum metal alloys, and not much work has been done experimentally since then on Pt-Rh, Ir-Rh, and Pt-Pd as we will show below.

Hence in this paper, a systematic study on alloy stability and ordering trends has been undertaken for these six alloys with a first-principles electronic structure approach. The configurational order has been formulated within the generalized perturbation method (GPM) [3-6]. The electronic properties of the reference medium on which the GPM relies were described in the framework of the self-consistent tight-binding-linear muffin-tin orbital (TBLMTO) multiple scattering formulation of the coherent potential approximation (CPA) [6,7].

Finally, temperature effects on local order and stability were accounted for by means of a standard generalized mean-field approach, namely the cluster variation method (CVM) $[4,8]$. From the present first-principles analysis, Pd-Rh, Pd-Ir and Pt-Ir exhibit clustering trends in full agreement with experimental observations. On the other hand, Pd-Pt, Rh-Ir and and Pt-Rh exhibit chemical order of the (1 00 ), (1 1/2 0 ) family-types, and a mixture of both, respectively (see Refs. [4,9] for a description of the structures). Because of the strength of the ordering tendencies, our theoretical analysis reveals that low-lying phase diagrams more complex than miscibility gap should exist for these three systems.

The paper is organized as follows. In section II, we review the results that are available 
in the literature, and comment on the salient features of the assessed phase diagrams of the six binary alloys. In section III, we discuss the electronic structure and equilibrium properties of the six alloys based on a first-principles TB-LMTO-CPA description of the electronic structure of disordered alloys. In section IV we present the results of the groundstate analysis of the Ising Hamiltonian based on a treatment of chemical order within the GPM. Finally, the results are summarized in section V.

\section{PHASE DIAGRAMS}

From the commonly accepted phase diagrams [1] shown in Fig. 1 for the six binary alloys made of $\mathrm{Rh}, \mathrm{Ir}, \mathrm{Pd}$, and $\mathrm{Pt}$, only a miscibility gap at low temperature characterizes the solid portion of the phase diagrams with maximum critical temperatures reported in Table I. At higher temperatures all these alloys form a series of completely miscible solid solutions based on the fcc lattice. However note that only three among the six miscibility gaps are reported with solid lines, indicating that only these three were actually based on experiments. Most of the original work on which these phase diagrams are based has been done by Raub in the late fifties [2]. According to Raub, a correlation could be made between the difference in the melting points of the alloy species and the highest critical temperature of the miscibility gap, at least from the experiments performed on Pd-Ir, Pt-Ir, and $\mathrm{Pd}-\mathrm{Rh}$, see Table I. Based solely on this observation, Raub goes on and "extrapolates a possible critical temperature for the miscibility gap of the Rh-Ir, Pd-Pt, and Pt-Rh alloys". These are basically the considerations (see below for further comments) on which the very hypothetical phase diagrams given in Ref. [1] are about! Let us now comment on each alloy phase diagram.

The phase diagram of the Pd-Rh has been described in Ref. [10]. The results have been confirmed by electrical resistivity measurements [11], and the thermodynamic mixing properties have been investigated to confirm the solid-state immiscibility [12-14].

The phase diagram of Pt-Rh redrawn in 1992 [15] with practically no input from exper- 
iment has been challenged in a recent study [16]. In this experimental work based on emf measurements the authors found a moderate negative deviation from Raoult's law for the activities, and relatively large negative enthalpies of mixing and negative excess entropy in the 900-1300 K temperature range. These results do not support the existence of a solidstate miscibility gap but instead point to the existence of some kind of ordering, and are in agreement with the shape of the solidus-liquidus lines. However no systematic characterization of the chemical order has been reported yet. It is worth noting that the author of the originally suggested phase diagram revisited the Pt-Rh system at a later time in a study on the ternary Au-Pt-Rh alloys, and concluded that long-time annealing of Pt-Rh samples for more than four years at $600{ }^{\circ} \mathrm{C}$ corroborated continuous solid solution with no initiation of decomposition [17]. Only recently a diffuse X-ray and small-angle neutron scattering analysis was performed on a Pt-47 at. \% Rh sample [18]. The results point to very "faint" ordering of the (1 1/2 0) family that seems to support the existence of a so-called "phase 40" [4,9] around equi-atomic composition with an estimated critical order-disorder temperature of about $185 \mathrm{~K}$.

The phase diagram of Pd-Ir originally investigated by Raub [2,19] in 1959 and 1964 has been revisited since then $[20,21]$. These experimental studies have confirmed the clustering trend in this system.

The phase diagram of Pt-Ir has also been originally proposed by Raub [2,22] and was based on experimental work. The phase diagram has been redrawn recently with a much higher maximum critical temperature of $1370{ }^{\circ} \mathrm{C}$ (instead of $975^{\circ} \mathrm{C}$ around 25 at.\% Ir) [23]. The positive departure from Raoult's law for the activities that was measured experimentally $[24,25]$ confirmed the clustering tendency in this system.

A recently assessed phase diagram of Rh-Ir has been proposed [26] on the basis of thermodynamic data [27] that seem to confirm the clustering trend in this system. However the highest critical temperature of the miscibility gap was set at $1335^{\circ} \mathrm{C}$ instead of the $850{ }^{\circ} \mathrm{C}$ suggested by Raub [2]. Although a large negative deviation from ideal solution is observed in this system, this is only an indication that a miscibility gap may exist since no direct 
measurement of short-range order (SRO) has been performed so far on this system.

Finally, the Pd-Pt phase diagram suggested by Raub [2] has been revisited in the seventies [28]. The results of vapor pressure by a torsion-effusion method, and of calorimetric heatof-solution measurements indicated a small negative deviation of the activities from ideal solution lines, moderately negative enthalpies of formation over the whole range of alloy composition, and positive entropies of formation smaller than the ideal entropies. These results have been confirmed by calorimetric investigation [29] and reviewed in Refs. [30,31]. Finally, X-ray diffuse scattering measurements have been performed in the late sixties [32]. Although this study concluded that SRO in Pd-Pt should exist, no detailed work has been yet reported to fully characterize it [33].

As said in Ref. [27], "a complete set of good thermodynamic data for all the binary systems discussed here would form a testing ground for the models of metallic phase stability". Here we will adopt the complementary view that theoretical analysis of this class of systems may motivate further thorough experimental work on these alloys by pointing out situations that may depart from commonly accepted or suggested propositions on alloy stability and chemical order.

\section{ELECTRONIC STRUCTURE MODELING AND EQUILIBRIUM PROPERTY RESULTS}

For the six chemically random fcc-based alloys electronic structure calculations were carried out on the basis of a charge self-consistent fully relativistic version of the tight-bindinglinear muffin-tin orbital-coherent potential approximation (TB-LMTO-CPA) method within the atomic sphere approximation (ASA) and the local density approximation (LDA) of density functional theory [6]. The LDA calculations were based on the exchange-correlation energy of Ceperley and Alder [34] as parameterized by Perdew and Zunger [35]. To eliminate the charge transfer effects, at each lattice parameter and alloy composition, the atomic sphere radii of the two species were adjusted in such a way that atoms were charge neutral 
while preserving the total volume of the alloy. The densities of states were evaluated on a line 0.005 Ry above the real axis (with an energy step of about $5 \mathrm{mRy}$ ) and then deconvoluted on the real axis. The CPA equations were solved iteratively using the method described in Ref. [6].

Calculations of the DOS, using the TB-LMTO-CPA method, have been performed as functions of composition for the six chemically random alloys based on the fcc lattice at their respective equilibrium lattice parameter. We show the DOS for the fcc-based pure elements in Fig. 2, and for the alloys at the three compositions $\mathrm{A}_{3} \mathrm{~B}, \mathrm{AB}$, and $\mathrm{AB}_{3}$ in Fig. 3. For the pure metals, the DOS are typical of the underlying fcc lattice, with a high DOS of $d$-character close to filled d-band. The number of $d$-electrons is about 8 for $R$ h and Ir, and 9 for $\mathrm{Pd}$ and $\mathrm{Pt}$, whereas the numbers of valence electrons are 9 and 10, respectively (cf. the plot of the integrated density of states versus energy in Fig. 2). These values are located on both sides of a high peak of electron density. In addition, one can expect stronger relativistic effects for the $5 \mathrm{~d}$ series (Ir and $\mathrm{Pt}$ ) than for the $4 \mathrm{~d}$ one ( $\mathrm{Rh}$ and $\mathrm{Pd}$ ) that tend to decrease the DOS at the Fermi energy, $n\left(E_{\mathrm{F}}\right)$, as shown in Fig. 2.

Since the difference in the number of valence electrons, $\Delta N_{\mathrm{v}}$ is unity in the case of X$\mathrm{Pd}$ and X-Pt (X=Rh, Ir), and zero for Rh-Ir and Pd-Pt, the scattering properties of the electrons vary very little in the alloys as functions of composition, and when compared to those of the pure elements. Indeed, as shown in Fig 3, the DOS are quite similar to those of the pure elements, especially in the case of Rh-Ir and Pd-Pt at equi-atomic composition. For these two alloys, $N_{\mathrm{v}}$ is constant and equal to the value associated with the species constituting the alloy. The alloying effect only causes a small smearing in the sharpness of the DOS of the alloy components. The partial DOS also displayed in Fig 3 are quite similar to those of the pure elements. Hence the main role of alloying is to fine-tune the location of the Fermi energy that is proportional to the average number of valence electrons, $N_{\mathrm{v}}$. The results of the DOS at the Fermi energy, $n\left(E_{\mathrm{F}}\right)$, reported in Fig. 4 are strongly indicative of what the variation of the electronic coefficient $\gamma$ that contributes a $\gamma T$-term to the heat capacity should be as a function of alloy composition, since [36] 


$$
\gamma=2 / 3 \pi^{2} k_{\mathrm{B}}^{2} n\left(E_{\mathrm{F}}\right)
$$

where $k_{\mathrm{B}}$ is Boltzmann's constant. However, for quantitative comparison of the predicted values of DOS at the Fermi energy to experimental results, an enhancement factor $(1+\lambda)$ has to be included in the r.h.s. of Eq. (3.1) to account for electron-phonon coupling. Note that since the d-band of $\mathrm{Pd}$ is completely below the Fermi level, see Fig. 2, and $E_{\mathrm{F}}$ is located in a region of high DOS, a substantial decrease in $\gamma$ can be expected when $\mathrm{Rh}$, Ir, or Pt solute is added to $\mathrm{Pd}$. In the case of the iso-electronic alloys, Rh-Ir and Pd-Pt, a similar trend in $\gamma$ is expected and reflects the transition from the $4 \mathrm{~d}$ to $5 \mathrm{~d}$ series that results in a moderate decrease in the DOS at the Fermi energy, as said before.

Based on TB-LMTO-CPA calculations the equilibrium properties have been obtained for the six chemically random alloys based on a fcc lattice, and the results are reported in Fig. 5 for the lattice parameter, and in Fig. 7 for the bulk modulus. As usually observed, the results that correspond to the actually observed structures are within about $1 \%$ for the lattice constant and 15\% for the bulk modulus. Note that for the fcc-based disordered alloys, a slight negative departure from Vegard's law is observed for all alloys in the entire range of composition, except for Pt-Rh and Pt-Ir. Usually, a positive (negative) departure from linearity is associated with a tendency towards phase decomposition (formation). However, here, since the departure is so insignificant very little can be inferred from the results, although this departure well reproduces the experimental data [17] as shown in Fig. 6 in the case of the Pt-Rh alloys. For the bulk modulus, see Fig. 7, there is a noticeable evolution with alloy composition since $B_{\mathrm{Pd}}<B_{\mathrm{Pt}} \sim B_{\mathrm{Rh}}<B_{\mathrm{Ir}}$, with Ir exhibiting the highest cohesive properties. Alloying effects beyond linear behavior with composition are here also very minor, except for Ir-Rh. For the pure metals, the theoretical values of 2.04, 2.68, 2.79, and 3.57 for the bulk modulus (in Mbar) of $\mathrm{Pd}, \mathrm{Pt}$, Rh, and Ir, respectively, compare well with 1.93 [37], 2.83 [38], 2.67 [39], and 3.73 [40], obtained experimentally. It should be noted that the evolution with alloy composition of the calculated bulk modulus (cf. Fig. 7), should translate in a similar variation of the Debye temperature, $\Theta_{\mathrm{D}}$ since both quantities are a 
good measure of the degree of cohesion in the solid phase. Since the Debye temperature is related to the coefficient $\beta$ that contributes a $\beta T^{3}$-term to heat capacity [36] according to

$$
\Theta_{\mathrm{D}}=(1.944 / \beta)^{1 / 2}
$$

the predictions presented here on the $\gamma$ and $\beta$ coefficients of specific heat should be fairly well confirmed experimentally.

\section{STABILITY AND ORDERING TRENDS}

Ordering trends have been predicted with the GPM $[3,4]$ in the context of the TB-LMTOCPA electronic structure description of the chemically disordered alloys. Within the GPM the ordering part of the total energy for a binary alloy $A_{1-c} B_{c}$ is given, at zero temperature and to second order in perturbation, by [3-5]

$$
\Delta E_{\mathrm{ord}}\left(\left\{q_{s}\right\}\right) \simeq \sum_{s} q_{s} V_{s}(c)
$$

with $q_{s}=c / 2\left(n_{s}^{\mathrm{BB}}-c n_{s}\right)$, where $n_{s}^{\mathrm{BB}}$ and $n_{s}$ refer to the number of $\mathrm{BB}$ pairs and the total number of pairs per site, respectively, associated with the $s^{\text {th }}$-neighbor shell, and $c$ is the concentration in B species. In this last equation, $V_{s}$ represents a $s^{\text {th }}$-neighbor effective pair interaction (EPI) given by $V_{s}=V_{s}^{\mathrm{AA}}+V_{s}^{\mathrm{BB}}-2 V_{s}^{\mathrm{AB}}$. Therefore, the sign convention is such that when $V_{s}>0(<0), \mathrm{AB}(\mathrm{AA}$ or $\mathrm{BB})$ pairs associated with a species at the origin and the other in the $s^{\text {th }}$-neighbor shell are favored. Within this formalism, the formation (or mixing) energy of the chemically random alloy is given by

$$
\Delta E_{\text {mix }}\left(\left\{c_{i}\right\}\right)=E_{\text {alloy }}^{\text {CPA }}-\sum_{i} c_{i} E_{i}^{\text {eq }}
$$

where $c_{i}$ is the concentration in species $i, E_{\text {alloy }}^{\mathrm{CPA}}$ and $E_{i}^{\mathrm{eq}}$ are the energies of the chemically random alloy and of the pure element $i$, respectively, at their corresponding equilibrium lattice parameter.

For practical purposes, this energy can be conveniently expressed by a so-called RedlichKister polynomial [41] as follows 


$$
\Delta E_{\text {mix }}\left(\left\{c_{i}\right\}\right)=c_{i} c_{j} \sum_{p}{ }^{p} L_{i j}\left(c_{i}-c_{j}\right)^{p}
$$

where by definition the two species $i$ and $j$ that form the alloy are in alphabetical order. For the six alloys studied the values of the three interaction parameters $\left\{{ }^{p} L ; p=[0,2]\right\}$ that enter Eq. (4.3) are reported in Table II.

The energy associated with a chemical configuration $\mathcal{C}$, defined by a set $\left\{q_{s}^{\mathcal{C}}\right\}$ at $0 \mathrm{~K}$, is given by

$$
\Delta E^{\mathcal{C}}\left(\left\{q_{s}\right\}\right)=\Delta E_{\text {mix }}\left(\left\{c_{i}\right\}\right)+\Delta E_{\text {ord }}\left(\left\{q_{s}^{\mathcal{C}}\right\}\right)
$$

Note that in the same way an ordering energy has been defined in the case of order, one can define a clustering (segregation) energy, $\Delta E_{\mathrm{Seg}}$, with the same expression as the one given by Eq. (4.1) but with $n_{s}^{\mathrm{BB}}=n_{s}$. However, the formation energy of the alloy will be approximately given by

$$
\Delta E_{\text {Form }} \sim-\Delta E_{\text {Seg }}
$$

The clustering energy is estimated from the concentration-weighted average of the DOS of the pure metals, each calculated at the average Fermi level of the average CPA medium, in the spirit of the GPM. On the other hand, $\Delta E_{\mathrm{Form}}$ is obtained from the contribution of the two sub-bands that are associated with each species and its own Fermi level. As long as the interfacial energies are negligible (which would be the case for alloy species exhibiting similar electron scattering properties, an assumption that is applicable to the present alloys), we expect the total energies that correspond to both situations (i.e., segregation within an alloy sample and juxtaposition of two metals) to be the same.

The variation of the first two EPIs, $V_{1}$ and $V_{2}$, with alloy composition is shown in Fig. 8 . The more distant EPIs are an order of magnitude lower than the first two, and therefore contribute little to the ordering energy (see below). These results can also be displayed as trajectories in a ground-state map spanned by the first- and second-nearest EPIs $[9,4]$ that are shaped by composition effect. From Fig. 9 and Table III, the clustering trends that are experimentally observed for $\mathrm{Rh}-\mathrm{Pd}, \mathrm{Ir}-\mathrm{Pd}$, and Ir-Pt are confirmed since in all three 
cases $V_{1}<0$ and $V_{2} / V_{1}<-1$. However, for the other three alloys, the situation is quite different. All three systems, Rh-Pt, Rh-Ir, and Pd-Pt exhibit ordering tendencies, contrary to suggestions made in the past, see section II. More specifically, for the Rh-Pt system, the $\left(\begin{array}{lll}1 & 0 & 0\end{array}\right)$ family of ordered states that consists of $\mathrm{L}_{2}$ for $\mathrm{Rh}_{3} \mathrm{Pt}$ and $\mathrm{RhPt}_{3}$, and $\mathrm{L}_{0}$ for $\mathrm{RhPt}$, is predicted. On the other hand Rh-Ir and Pd-Pt should display ordered states of the (1 1/2 0) family, i.e., $\mathrm{DO}_{22}$ at the $\mathrm{A}_{3} \mathrm{~B}$ and $\mathrm{AB}_{3}$ compositions, $\mathrm{C}_{1} 1_{\mathrm{b}}$ order (of $\mathrm{Pt}_{2}$ Mo type) at the compositions $\mathrm{A}_{2} \mathrm{~B}$ and $\mathrm{AB}_{2}$, "phase 40 " [9,4] at equi-atomic composition, and finally $\mathrm{A}_{5} \mathrm{~B}$-type of order [9]. It is interesting to note that in the case of Rh-Pt, the mixing energy is slightly positive whereas in the case of Ir-Pd the mixing energy is slightly negative although the former has a clear tendency to order whereas the later displays a definite tendency towards phase separation.

By including in the ground-state analysis EPIs up to the fourth neighbor distance, the results are unchanged except for $\mathrm{Pd}-\mathrm{Pt}$ that should exhibit order of the (1 00 ) family, as indicated in Table IV. Note also that in the case of Rh-Pt below 50 at.\% Rh the ordering trends switch from the $\left(\begin{array}{lll}1 / 2 & 0\end{array}\right)$ to the $\left(\begin{array}{lll}1 & 0 & 0\end{array}\right)$ family of ordered states.

The expected ground states together with the values of the EPIs and the use of Eq. (4.1) allow us to predict the magnitude of the ordering energies that controls the shape of the phase diagram at finite temperature. The combined variation of the mixing energy, see Eqs. (4.2) and (4.3), and of the ordering energy, Eq. (4.1), with alloy composition provides the phase diagram of each alloy at $T=0 \mathrm{~K}$, as shown in Fig. 10. From this study it can be concluded that as observed experimentally, the three alloys, $\mathrm{Rh}-\mathrm{Pd}$, Ir-Pd, and Ir-Pt should definitely phase separate, in accordance with experimental facts. On the other hand, Rh-Ir and Pd-Pt should exhibit all the ordered states that belong to the ( $\left.\begin{array}{lll}1 & 1 / 2 & 0\end{array}\right)$ - and $\left(\begin{array}{lll}1 & 0 & 0\end{array}\right)-$ families of order, respectively. Finally, in the case of Rh-Pt, despite a slightly positive mixing energy, this system should exhibit a $\mathrm{D}_{22}$ ordered state and the so-called "phase 40 " [9] at the compositions $\mathrm{Rh}_{3} \mathrm{Pt}$ and $\mathrm{RhPt}$, respectively.

Our predictions are in qualitative agreement with those obtained for all six systems based on the calculation of the total energy for some alloy configurations carried out with 
an "exact" muffin-tin orbital method (EMTO) within the LDA or the generalized gradient approximation (GGA) of density functional theory [42]. For the sake of quantitative comparison, we also report in Table IV the formation energies of Ref. [43] for Pd-Pt, Rh-Pd, and Rh-Pt alloys. These results were obtained from the application of the semi-relativistic linearized augmented plane-wave (LAPW) method [44]. Considering that our approach is solely based on the knowledge of the properties of the random state of an alloy, our results compare favorably in a quantitative sense with those of Ref. [43]. Our predictions obtained for $\mathrm{Pd}-\mathrm{Pt}$ and $\mathrm{Pt}-\mathrm{Rh}$ are also in qualitative agreement with those based on recent ultra-soft pseudo-potential LDA and projector-augmented wave GGA calculations [45]. For both alloys, the latter results also predict the existence of ordered structures not yet seen experimentally. In the case of $\mathrm{Pd}-\mathrm{Pt}$ alloys, $\mathrm{Pd}_{3} \mathrm{Pt}$ and $\mathrm{PdPt}_{3}$ prototype ordered structures, and a $\mathrm{L} 1_{1} \mathrm{PdPt}$, close in energy to $\mathrm{L} 1_{0}$, have been found with small heats of formation not exceeding $-3 \mathrm{mRy} /$ atom. In the case of $\mathrm{Pt}-\mathrm{Rh}$ alloys, $\mathrm{D} 1_{\mathrm{a}} \mathrm{Pt}_{4} \mathrm{Rh}$ and $\mathrm{PtRh}_{4}, \mathrm{D0}_{22} \mathrm{Pt}_{3} \mathrm{Rh}$ and $\mathrm{PtRh}_{3}, \mathrm{C} 49 \mathrm{PtRh}_{2}$, and ordered PtRh were predicted with a minimum heat of formation of about $-1.8 \mathrm{mRy} /$ atom found for PtRh.

Despite these predictions on chemical order in Rh-Ir, Pd-Pt, and Pt-Rh, it may be difficult to characterize long-range order (LRO), and even to identify SRO with X-ray or neutron diffuse scattering experiments. Indeed, the ordering trends that have been predicted in this work are rather weak, and the ordering energies themselves are less than $2 \mathrm{mRy} / \mathrm{atom}$, see Table IV. In addition, since all the order-disorder transitions that would be involved are first-order, the SRO is expected to decrease rapidly with temperature above the low-lying critical order-disorder transition. Altogether, it means that thermal annealing of samples at temperatures where SRO should still exist may not be possible or takes a very long time due to slow diffusion kinetics. A recent diffuse X-ray and small-angle neutron scattering analysis performed on a Pt 47 at.\% Rh sample revealed very weak maxima of diffuse intensity in the (1 $1 / 20)$ positions in reciprocal space that are associated with the existence of a "phase 40" [18]. The extraction of the EPIs by an inverse Monte Carlo method led to an estimate of the critical order-disorder temperature of about $185 \mathrm{~K}$, a lower bound according to the 
authors of this work since relaxation-time effect may cause the actual magnitude of the EPIs to slightly increase. It is worth noting that the first two EPIs of 2.35 and $-0.41 \mathrm{mRy} /$ atom obtained experimentally with the Borie-Sparks separation technique [18] compare favorably with our theoretical values of 3.73 and $-0.28 \mathrm{mRy} /$ atom for a $\mathrm{Pt}_{50} \mathrm{Rh}_{50}$ alloy, cf. Fig. 11. This figure also confirms the rapid convergence of the EPIs with distance, beyond the second nearest neighbor distance.

To examine temperature effects on ordering trends and tie them to phase diagrams, critical order-disorder temperatures were estimated with the generalized mean-field CVM $[4,8]$ within the tetrahedron-octahedron approximation. This allowed us to properly describe the ordering contribution to the Gibbs energy up to the second nearest EPI as well as the configurational entropy as functions of temperature. The more distant interactions, third and fourth EPIs, were also accounted for in the ordering energy with a Bragg-Williams treatment. For the three alloys, Rh-Ir, Pt-Rh, and Pd-Pt at equi-atomic composition, the critical order-disorder temperatures are $246 \mathrm{~K}$ ("40" $\rightarrow$ Dis), $325 \mathrm{~K}$ ("40" $\rightarrow$ Dis), and $422 \mathrm{~K}$ $\left(\mathrm{L} 1_{0} \rightarrow\right.$ Dis), respectively. These low values just reflect the weak ordering tendencies found for this class of alloys.

Finally, let us review the points that could help us rationalize stability and ordering trends that we found for these six substitutional alloys in the context of electronic structure properties. On one hand, for the two iso-electronic systems, Rh-Ir and Pd-Pt, that involve elements from the $4 \mathrm{~d}$ and $5 \mathrm{~d}$ series, a definite and similar tendency towards order is predicted. It is interesting to note that a standard d-band tight-binding (TB) analysis (with only diagonal-disorder effect included) of ordering for these two alloys would predict a clustering tendency since the average numbers of valence d-electrons are 8 and 9 electrons, respectively [4]. The trend from $a b$ initio can be attributed to $s$ - $d$ hybridization, and to relavistic effects that cannot be ignored for elements of the $5 \mathrm{~d}$ series. On the other hand for the two alloys that exhibit a $\Delta N_{\mathrm{v}}$ of unity with elements belonging to the same series ( $4 \mathrm{~d}$ or $\left.5 \mathrm{~d}\right)$, i.e. for Rh-Pd and Ir-Pt, a tendency towards phase separation is predicted, in accordance with the d-band TB analysis since, in this case, $s-d$ hybridization and relativistic effect cancel out, 
in accordance with the assumption made in a standard TB analysis. For these first two classes of systems, the alloys are located in a similar region of Fig. 9. Finally for the last two alloys that mix elements belonging to the $4 \mathrm{~d}$ and $5 \mathrm{~d}$ series with a $\Delta N_{\mathrm{v}}$ of unity, i.e. Rh-Pt and Pd-Ir, a mixed situation is encountered. Indeed, whereas the sign of the mixing energy indicates favorable (unfavorable) phase formation for Ir-Pd (Rh-Pt), the ordering trends indicate the opposite, i.e. phase separation for Ir-Pd and order for Rh-Pt. This ambiguity can be lifted if one notes that the difference in d-band widths, $\Delta W_{\mathrm{d}}$, of the pure elements is about 0.015 Ry for Rh-Pt whereas it is 0.250 Ry for Ir-Pd (for the other four alloys this difference is $0.140,0.125,0.110$, and 0.125 Ry for Rh-Ir, Pd-Pt, Rh-Pd, and Ir-Pt, respectively) [46]. Since $\Delta W_{\mathrm{d}}$ is a measure of the off-diagonal disorder in the TB language, a large value of this quantity favors clustering. This would explain why Ir-Pd displays a tendency towards phase separation whereas Rh-Pt shows a tendency towards order. Hence for all six alloy systems studied, the combined effects of $s-d$ hybridization, relativity, and off-diagonal disorder lead to the definition of three classes of alloys. It should be noted that here $N_{\mathrm{v}}$ has little effect on the chemical trends, and only a small impact on the magnitude of the mixing energy. The same can be said of $\Delta N_{\mathrm{v}}$ that is a measure of the difference in the scattering properties of the electrons that control the strength of stability. Here $\Delta N_{\mathrm{v}} \leq 1$, and therefore this leads overall to weak magnitudes for the mixing energies and the ordering (or segregation) energies for the six alloy systems.

It is worth mentioning that based on these trends, and the arguments put forward to explain them, the ordering tendency predicted and observed in the Ni-Pt system should not come as a surprise. Indeed, the Pt-Pd system belongs to the $\left(\begin{array}{lll}1 & 0 & 0\end{array}\right)$-family of order, and the same is true for Ni-Pt. Despite the theoretical controversy that arose about this system [47], the diagonal disorder parameter, $\Delta W_{\mathrm{d}}=0.243 \mathrm{Ry}$, relativistic effects that particularly apply to Pt and its alloys, and $s-d$ hybridization are all responsible for the chemical order observed in this iso-electronic alloy. 


\section{CONCLUSIONS}

An $a b$ initio alloy theory has been applied to the study of the relation between constitution (phase) diagrams, electronic structure, and stability and ordering properties for the binary systems made of the four fcc-based transition metals Rh, Ir, Pd, and Pt. For three of the six binary alloys, namely Rh-Pd, Ir-Pd, and Ir-Pt, the phase separation trend that was observed experimentally is fully confirmed by our calculations. On the other hand, for the other three binary alloys, Rh-Pt, Rh-Ir, and Pd-Pt, ordering trends are predicted, in contradiction with suggested and accepted phase separation tendencies. For Pd-Pt, Rh-Ir,

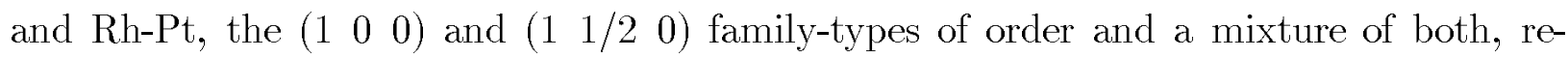
spectively, are predicted. The impact of relativistic effects and $s$ - $d$ hybridization explains

to some extent the ordering trends that are predicted for these three alloys, a finding that could not be anticipated on the basis of semi-phenomenological tight-binding calculations that only rely on d-band analysis. The predictions made on the variation with alloy composition of the electron and phonon contributions to specific heat, if confirmed experimentally, should validate the electronic structure description of these alloys. Finally, detailed analysis of short-range order from diffuse scattering measurements should validate the ordering trends discussed here. If confirmed, these predictions should impact the way these alloys and the higher-order component alloys that contain them are considered in a thermodynamic analysis, even at high temperature since short-range order has important consequences on physical properties.

\section{ACKNOWLEDGMENTS}

The work of P. T. has been performed under the auspices of the U.S. Department of Energy by the University of California Lawrence Livermore National Laboratory under contract No. W-7405-ENG-48. The work of V. D. and J. K. was carried out within the Project No. AV0Z1-010-914 of the Academy of Sciences of the Czech Republic, and supported by 
the Grant Agency of the Academy of Sciences of the Czech Republic under Project No. AVOZ10100520. 


\section{REFERENCES}

[1] Binary Alloy Phase Diagrams, T. B. Massalski ed. (ASM International, Materials Park, OH, 1990), vols. 1 to $\mathbf{3}$.

[2] E. Raub, J. Less-Com. Met. 1 (1), 3 (1959).

[3] F. Ducastelle and F. Gautier, J. Phys. F: Met. Phys. 6, 2039 (1976).

[4] F. Ducastelle, Order and Phase Stability in Alloys, ed. by F. R. de Boer and D. G. Pettifor (North-Holland, Amsterdam, 1991), Cohesion and Structure series, vol. 3.

[5] P. E. A. Turchi, in Intermetallic Compounds: Principles and Practice, ed. by J. H. Westbrook and R. L. Fleischer (John Wiley \& Sons, Ltd., New York, 1995), vol. 1, Chap. 2, pp. 21-54.

[6] I. Turek, V. Drchal, J. Kudrnovský, M. Šob, and P. Weinberger, Electronic Structure of Disordered Alloys, Surfaces and Interfaces (Kluwer, Boston-London-Dordrecht, 1997).

[7] J. S. Faulkner, in Progress in Materials Science, ed. by J. W. Christian, P. Hassen and T. B. Massalski (Pergamon Press, New York, 1982), vol. 27, No. 1 and No. 2; and references cited therein.

[8] R. Kikuchi, Phys. Rev. 81, 988 (1951).

[9] J. Kanamori and Y. Kakehashi, J. Phys. Colloq. (Paris) 38, C7-274 (1977).

[10] S. N. Tripathi and S. R. Bharadwaj, J. of Phase Equil. 15 (2), 208 (1994); and references therein.

[11] J. E. Shield and R. K. Williams, Scripta Metall, 21, 1475 (1987).

[12] K. M. Myles, Trans. of the Metall. Soc. of AIME 242, 1523 (1968).

[13] R. Gürler, L. A. Cornish, and J. N. Pratt, J. of Alloys and Comp. 191, 165 (1993).

[14] K. T. Jacob, S. Priya, and Y. Waseda, J. of Phase Equil. 19 (4), 340 (1998). 
[15] H. Okamoto, J. of Phase Equil. 13 (2), 223 (1992).

[16] K. T. Jacob, S. Priya, and Y. Waseda, Metall. and Mater. Trans. 29A, 1545 (1998).

[17] E. Raub and G. Kalkenburg, Z. Metallkde 55, 392 (1964).

[18] Ch. Steiner, B. Schönfeld, M. J. Portmann, M. Kompatscher, G. Kostorz, A. Mazuelas, T. Metzger, J. Kohlbrecher, and B. Demé, Phys. Rev. B 71, 104204 (2005).

[19] E. Raub and E. Röschel, Z. Metallkde. 55, 320 (1964).

[20] S. N. Tripathi, S. R. Bharadwaj, and M. S. Chandrasekharaiah, J. of Phase Equil. 12 (5), 603 (1991); and references therein.

[21] Y. I. Vesnin, Y. V. Shubin, and S. V. Korenev, Russian J. of Phys. Chem. 70 (2), 217 (1996).

[22] E. Raub and W. Plate, Z. Metallkde. 47, 688 (1956).

[23] S. R. Bharadwaj, S. N. Tripathi, and M. S. Chandrasekharaiah, J. of Phase Equil. 16 (5), 460 (1995); and references therein.

[24] E. S. Ramkrishnan and M. S. Chandrasekharaiah, J. Less-Com. Met. 37, 269 (1974).

[25] S. N. Tripathi and M. S. Chandrasekharaiah, J. Less-Com. Met. 91, 251 (1983).

[26] S. N. Tripathi, S. R. Bharadwaj, and M. S. Chandrasekharaiah, J. of Phase Equil. 12 (5), 606 (1991); and references therein.

[27] S. N. Tripathi and M. S. Chandrasekharaiah, Z. Metallkde 74 (4), 241 (1983).

[28] J. B. Darby, Jr. and K. M. Myles, Metall. Trans. 3, 653 (1972).

[29] F. H. Hayes and O. Kubaschewski, Metal Sci. J. 5, 37 (1971).

[30] S. R. Bharadwaj and S. N. Tripathi, J. Alloy Phase Diag. 6, 118 (1990); and references therein. 
[31] S. R. Bharadwaj, A. S. Kerbar, S N. Tripathi, and S. R. Dharwadkar, J. Less-Com. Met. 169 (1), 167 (1991); and references therein.

[32] A. Kidron, Phys. Lett. 25A, 112 (1967).

[33] H. Noh, T. B. Flanagan, and Y. Sakamoto, Scripta Metall. 29, 445 (1993).

[34] D. M. Ceperley and B. J. Alder, Phys. Rev. Lett. 45, 566 (1980).

[35] J. P. Perdew and A. Zunger, Phys. Rev. B 23, 5048 (1981).

[36] C. Kittel, Introduction to Solid State Physics, (John Wiley Sons, New York, 1971), Fourth Edition.

[37] J. A. Rayne, Phys. Rev. 118, 1545 (1960).

[38] R. E. MacFarlane, Phys. Lett. 18, 91 (1965).

[39] E. Walker, J. Ashkenazi, and M. Dacorogna, Phys. Rev. B 24, 2254 (1981).

[40] R. E. MacFarlane, J. A. Rayne, and C. K. Jones, Phys. Lett. 20, 234 (1966).

[41] O. Redlich and A. Kister, Ind. Eng. Chem. 40, 345 (1948).

[42] H. L. Skriver, Materials Science Databases, http://databases.fysik.dtu.dk/.

[43] Z. W. Lu, S.-H. Wei, and A. Zunger, Phys. Rev. Lett. 66, 1753 (1991); Z. W. Lu, B. M. Klein, and A. Zunger, J. Phase Equil. 16 (1), 36 (1995).

[44] S.-H. Wei and H. Krakauer, Phys. Rev. Lett. 55, 1200 (1985); S.-H. Wei, H. Krakauer, and M. Weinert, Phys. Rev. B 32, 7792 (1985); and references therein.

[45] S. Curtarolo, D. Morgan, and G. Ceder, CALPHAD 29, 163 (2005).

[46] O. K. Andersen, O. Jepsen, and D. Glötzel, in Highlights of Condensed Matter Theory, ed. by F. Bassani, F. Fumi, and M. P. Tosi (North-Holland, New York, 1985), pp. 59.

[47] P. P. Singh, A. Gonis, and P. E. A. Turchi, Phys. Rev. Lett. 71, 1605 (1993); and 
references therein. 


\section{TABLES}

TABLE I. For each A-B alloys, the table indicates the number of valence electrons for each species $i, N_{i}$, and the difference in the number of valence electrons, $\Delta N_{\mathrm{v}}=N_{\mathrm{B}}-N_{\mathrm{A}}$, the maximum value of the temperature of the miscibility gap, $T_{\mathrm{MG}}$, and the melting point of each species $i, T_{\mathrm{M}}^{i}$. Temperatures are given in ${ }^{\circ} \mathrm{C}$.

\begin{tabular}{|c|c|c|c|c|c|c|}
\hline A-B Alloy & $N_{\mathrm{A}}$ & $N_{\mathrm{B}}$ & $\Delta N_{\mathrm{v}}$ & $T_{\mathrm{MG}}$ & $T_{\mathrm{M}}^{\mathrm{A}}$ & $T_{\mathrm{M}}^{\mathrm{B}}$ \\
\hline Rh-Ir & 9 & 9 & 0 & 850 & 2447 & 1963 \\
\hline $\mathrm{Pd}-\mathrm{Pt}$ & 10 & 10 & 0 & 770 & 1555 & 1769 \\
\hline $\mathrm{Rh}-\mathrm{Pd}$ & 9 & 10 & 1 & 845 & 1963 & 1555 \\
\hline $\mathrm{Rh}-\mathrm{Pt}$ & 9 & 10 & 1 & 760 & 1963 & 1769 \\
\hline $\mathrm{Ir}-\mathrm{Pd}$ & 9 & 10 & 1 & 1482 & 2447 & 1555 \\
\hline $\mathrm{Ir}-\mathrm{Pt}$ & 9 & 10 & 1 & 975 & 2447 & 1769 \\
\hline
\end{tabular}

TABLE II. Three-term Redlich-Kister analysis of the mixing energy (in mRy/atom) for each of the six fcc-based alloys.

\begin{tabular}{cccc}
\hline \hline A-B Alloy & ${ }^{0} L$ & ${ }^{1} L$ & ${ }^{2} L$ \\
\hline Ir-Rh & -17.97915 & -1.02461 & -0.21934 \\
Pd-Pt & -15.64755 & -0.48400 & -0.09801 \\
Pd-Rh & +16.37361 & -0.63817 & -0.23707 \\
Pt-Rh & +1.90672 & +1.40107 & -0.97414 \\
Ir-Pd & -2.03042 & -0.82296 & -1.66072 \\
Ir-Pt & +18.63975 & +0.74137 & +0.37826 \\
\hline \hline
\end{tabular}


TABLE III. Ground-state analysis up to the second nearest-neighbor EPI for the six fcc-based alloys. For each ordered state the ordering and formation (in parenthesis) energies are given in mRy/atom. The ordering energy associated with phase separation (PS; see text) is also indicated for all alloys at equi-atomic composition.

\begin{tabular}{|c|c|c|c|c|c|c|}
\hline A-B & Rh-Ir & $\mathrm{Pd}-\mathrm{Pt}$ & Rh-Pd & Rh-Pt & $\mathrm{Ir}-\mathrm{Pd}$ & $\mathrm{Ir}-\mathrm{Pt}$ \\
\hline $\mathrm{A}_{5} \mathrm{~B}$ & $-0.37(-2.78)$ & $-0.51(-2.74)$ & $0.82(2.98)$ & $-0.74(-0.40)$ & $1.29(0.86)$ & $0.71(3.37)$ \\
\hline $\mathrm{L}_{2}\left(\mathrm{~A}_{3} \mathrm{~B}\right)$ & $-0.52(-3.82)$ & $-0.81(-3.79)$ & $1.42(4.55)$ & $-1.73(-1.54)$ & $3.08(2.55)$ & $1.46(5.02)$ \\
\hline $\mathrm{D0}_{22}\left(\mathrm{~A}_{3} \mathrm{~B}\right)$ & $-0.62(-3.92)$ & $-0.90(-3.88)$ & $1.54(4.66)$ & $-1.68(-1.49)$ & $3.02(2.49)$ & $1.52(5.08)$ \\
\hline $\mathrm{C} 11_{\mathrm{b}}\left(\mathrm{A}_{2} \mathrm{~B}\right)$ & $-0.68(-4.57)$ & $-0.88(-4.37)$ & $1.52(5.17)$ & $-1.37(-1.07)$ & $2.62(+2.05)$ & $1.46(+5.64)$ \\
\hline $\mathrm{L} 1_{0}(\mathrm{AB})$ & $-0.80(-5.28)$ & $-1.01(-4.92)$ & $2.01(6.11)$ & $-2.08(-1.61)$ & $4.17(3.66)$ & $2.06(6.73)$ \\
\hline $40\left(\mathrm{~A}_{2} \mathrm{~B}_{2}\right)$ & $-0.97(-5.46)$ & $-1.19(-5.10)$ & $2.22(6.31)$ & $-1.94(-1.47)$ & $4.02(3.51)$ & $2.17(6.83)$ \\
\hline $\mathrm{L}_{1}(\mathrm{AB})$ & $-0.27(-4.75)$ & $-0.26(-4.17)$ & $0.31(4.40)$ & $0.21(0.68)$ & $-0.22(-0.73)$ & $0.16(4.82)$ \\
\hline PS (AB) & 3.45 & 4.08 & -7.28 & 5.39 & -11.62 & -6.83 \\
\hline $\mathrm{C} 11_{\mathbf{b}}\left(\mathrm{AB}_{2}\right)$ & $-0.73(-4.80)$ & $-0.83(-4.26)$ & $1.58(5.15)$ & $-1.15(-0.66)$ & $2.62(2.21)$ & $1.48(5.56)$ \\
\hline $\mathrm{L}_{2}\left(\mathrm{AB}_{3}\right)$ & $-0.68(-4.15)$ & $-0.74(-3.63)$ & $1.61(4.61)$ & $-1.44(-0.98)$ & $2.96(2.58)$ & $1.54(4.98)$ \\
\hline $\mathrm{DO}_{22}\left(\mathrm{AB}_{3}\right)$ & $-0.75(-4.22)$ & $-0.82(-3.71)$ & $1.69(4.69)$ & $-1.36(-0.91)$ & $2.93(2.55)$ & $1.59(5.04)$ \\
\hline $\mathrm{AB}_{5}$ & $-0.396(-3.00)$ & $-0.44(-2.57)$ & $0.75(2.65)$ & $-0.52(-0.61)$ & $1.14(0.85)$ & $0.74(3.23)$ \\
\hline
\end{tabular}


TABLE IV. Ground-state analysis up to the fourth nearest-neighbor EPI for the six fcc-based alloys. For each ordered state the ordering and formation (in parenthesis) energies are given in mRy/atom. The ordering energy associated with phase separation (PS; see text) is also indicated for all alloys at equi-atomic composition. For comparison purposes, a second line indicates the formation energy from Ref. [43] whenever available.

\begin{tabular}{|c|c|c|c|c|c|c|}
\hline A-B & Rh-Ir & Pd-Pt & Rh-Pd & Rh-Pt & Ir-Pd & $\mathrm{Ir}-\mathrm{Pt}$ \\
\hline $\mathrm{A}_{5} \mathrm{~B}$ & $-0.46(-2.88)$ & $-0.59(-2.82)$ & $0.53(2.69)$ & $-0.74(-0.41)$ & $0.75(0.32)$ & $0.49(3.15)$ \\
\hline \multirow[t]{2}{*}{$\mathrm{L}_{2}\left(\mathrm{~A}_{3} \mathrm{~B}\right)$} & $-0.32(-3.62)$ & $-1.33(-4.32)$ & $2.48(5.61)$ & $-1.55(-1.36)$ & $4.45(3.92)$ & $2.86(6.42)$ \\
\hline & & $(-2.23)$ & $(6.25)$ & $(-1.50)$ & & \\
\hline \multirow[t]{2}{*}{$\mathrm{D}_{22}\left(\mathrm{~A}_{3} \mathrm{~B}\right)$} & $-0.70(-3.99)$ & $-1.02(-4.00)$ & $1.40(4.53)$ & $-1.67(-1.48)$ & $2.62(2.08)$ & $1.45(5.00)$ \\
\hline & & $(-1.62)$ & $(4.69)$ & $(-1.85)$ & & \\
\hline \multirow[t]{2}{*}{$\mathrm{C} 11_{\mathrm{b}}\left(\mathrm{A}_{2} \mathrm{~B}\right)$} & $-0.86(-4.75)$ & $-0.78(-4.28)$ & $0.89(4.54)$ & $-1.45(-1.15)$ & $1.50(0.93)$ & $0.75(4.93)$ \\
\hline & & $(-2.07)$ & $(4.72)$ & $(-0.74)$ & & \\
\hline \multirow[t]{2}{*}{$\mathrm{L} 1_{0}(\mathrm{AB})$} & $-0.56(-5.05)$ & $-1.80(-5.71)$ & $3.00(7.09)$ & $-1.94(-1.47)$ & $5.56(5.05)$ & $3.50(8.16)$ \\
\hline & & $(-2.90)$ & $(6.06)$ & $(-1.21)$ & & \\
\hline \multirow[t]{2}{*}{$40\left(\mathrm{~A}_{2} \mathrm{~B}_{2}\right)$} & $-1.23(-5.72)$ & $-0.97(-4.88)$ & $1.35(5.45)$ & $-2.04(-1.58)$ & $2.37(1.87)$ & $1.17(5.83)$ \\
\hline & & $(-2.36)$ & $(5.20)$ & $(-1.21)$ & & \\
\hline \multirow[t]{2}{*}{$\mathrm{L}_{1}(\mathrm{AB})$} & $-0.31(-4.80)$ & $-1.11(-5.03)$ & $0.49(4.58)$ & $0.26(0.73)$ & $-0.60(-1.11)$ & $0.81(5.47)$ \\
\hline & & $(-2.14)$ & $(5.81)$ & $(-0.29)$ & & \\
\hline PS (AB) & 2.58 & 3.01 & -9.53 & 5.15 & -17.32 & -8.56 \\
\hline \multirow[t]{2}{*}{$\mathrm{C} 11_{\mathbf{b}}\left(\mathrm{AB}_{2}\right)$} & $-0.89(-4.96)$ & $-0.83(-4.26)$ & $1.03(4.60)$ & $-1.15(-0.66)$ & $1.51(1.11)$ & $0.84(4.92)$ \\
\hline & & $(-2.23)$ & $(5.33)$ & $(-0.32)$ & & \\
\hline \multirow[t]{2}{*}{$\mathrm{L}_{2}\left(\mathrm{AB}_{3}\right)$} & $-0.53(-4.01)$ & $-1.36(-4.25)$ & $2.18(5.17)$ & $-1.43(-0.98)$ & $3.91(3.53)$ & $2.40(5.85)$ \\
\hline & & $(-2.58)$ & $(4.91)$ & $(-0.79)$ & & \\
\hline \multirow[t]{2}{*}{$\mathrm{D}_{22}\left(\mathrm{AB}_{3}\right)$} & $-0.82(-4.29)$ & $-0.88(-3.77)$ & $1.61(4.60)$ & $-1.39(-0.94)$ & $2.41(2.03)$ & $1.41(4.85)$ \\
\hline & & $(-2.14)$ & $(5.07)$ & $(-0.96)$ & & \\
\hline $\mathrm{AB}_{5}$ & $-0.48(-3.08)$ & $-0.42(-2.55)$ & $0.52(2.42)$ & $-0.52(-0.61)$ & $0.51(0.22)$ & $0.46(2.95)$ \\
\hline
\end{tabular}




\section{FIGURES}

FIG. 1. (Color online) Assessed phase diagrams of the Rh-Ir, Pd-Pt, Rh-Pd, Ir-Pt, Rh-Pt, and Ir-Pd binary alloy systems, taken from Ref. [1].

FIG. 2. DOS of the pure elements (solid line) Rh, Pd, Ir, and Pt based on the fcc structure. The dotted line indicates the variation of the integrated density of states with energy. The Fermi energy $\mathrm{E}_{\mathrm{F}}$ is indicated by a vertical line and taken as zero of energy.

FIG. 3. Total (solid line) and partial (dotted and dashed lines) DOS of fcc-based $\mathrm{A}_{3} \mathrm{~B}$ (left panels), $\mathrm{AB}$ (central panels), and $\mathrm{AB}_{3}$ (right panels) alloys. The Fermi energy $\mathrm{E}_{\mathrm{F}}$ is indicated by a vertical line and taken as zero of energy.

FIG. 4. Variation of the DOS at the Fermi energy, $n\left(E_{\mathrm{F}}\right)$, as a function of alloy composition for the six fcc-based alloys Pd-Rh, Pd-Ir, Pt-Rh, Pt-Ir, Ir-Rh, and Pd-Pt.

FIG. 5. Variation of the lattice constant (in $\AA$ ) for the six fcc- based alloys as a function of composition.

FIG. 6. Variation of the departure of the lattice constant from Vegard's law, $\delta a=a_{\text {alloy }}^{\text {eq }}-\sum_{i=\mathrm{A}, \mathrm{B}} c_{i} a_{i}^{\mathrm{eq}}\left(\mathrm{x} 10^{3}\right.$, in $\left.\AA\right)$, with Rh composition in the case of Pt-Rh alloys. The solid line corresponds to the theoretical results and the dotted line to the experimental data from Ref. [17].

FIG. 7. Variation of the bulk modulus (in Mbar) for the six fcc-based alloys as a function of composition.

FIG. 8. Variation of the first and second nearest-neighbor effective pair interactions (in mRy/atom) for the six fcc-based alloys with composition.

FIG. 9. Variation of the effective pair interactions with alloy composition in a ground-state map representation (first versus second effective pair interaction) for the six fcc-based alloys. For each trajectory, the filled square corresponds to the null composition of the underlined element. 
FIG. 10. Formation energy of the disordered state as a function of alloy composition (dotted line) for the six fcc-based alloys. Configuration energies of the most likely ground states (full circles), including minus the energies of the segregated state (solid curve, when applicable) have also been reported.

FIG. 11. Variation of the effective pair interaction (in mRy/atom) with distance (in units of the equilibrium fcc lattice parameter a) for a $\mathrm{Pt}_{50} \mathrm{Rh}_{50}$ alloy. 

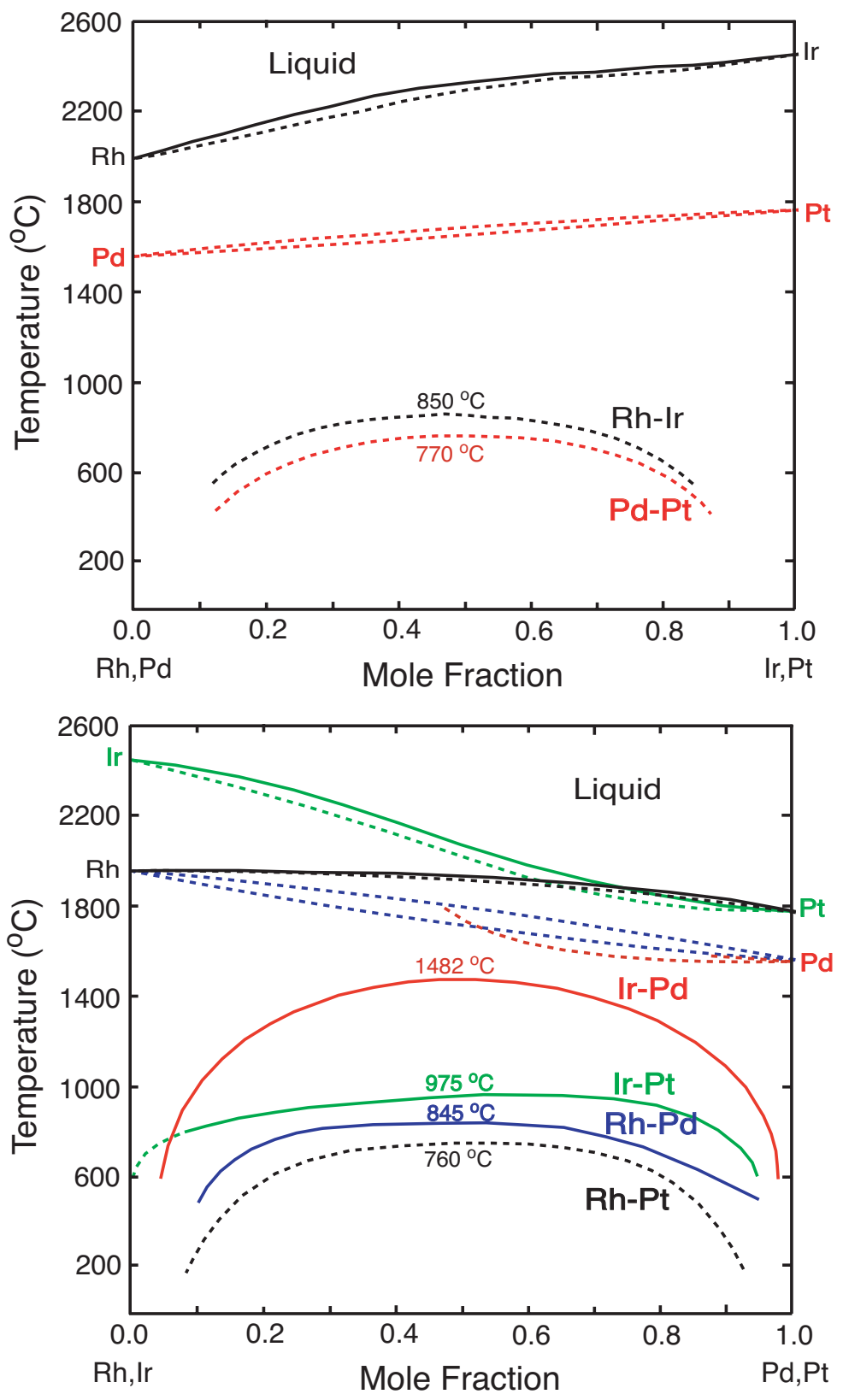

FIG. 1. Turchi et al. 

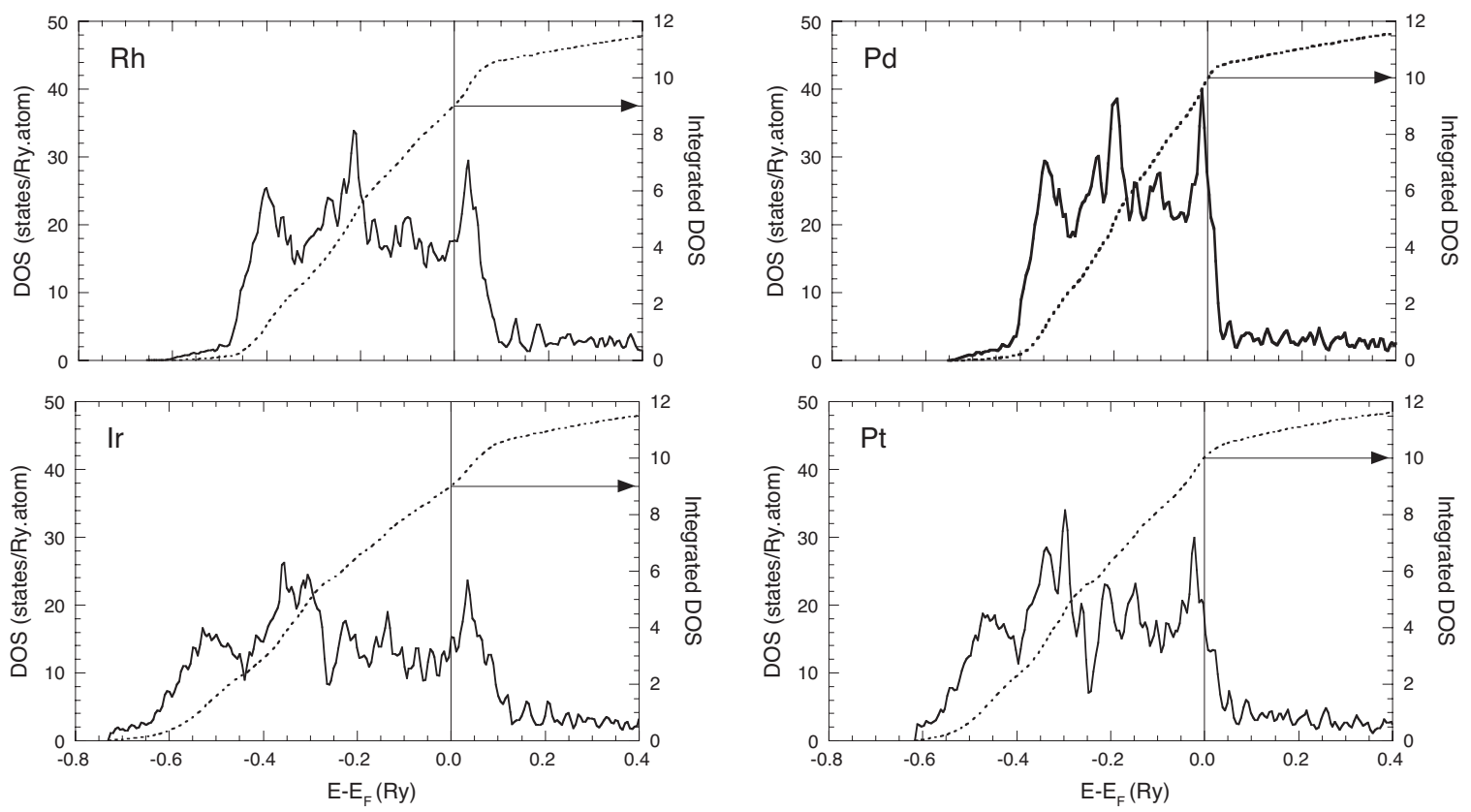

FIG. 2. Turchi et al. 

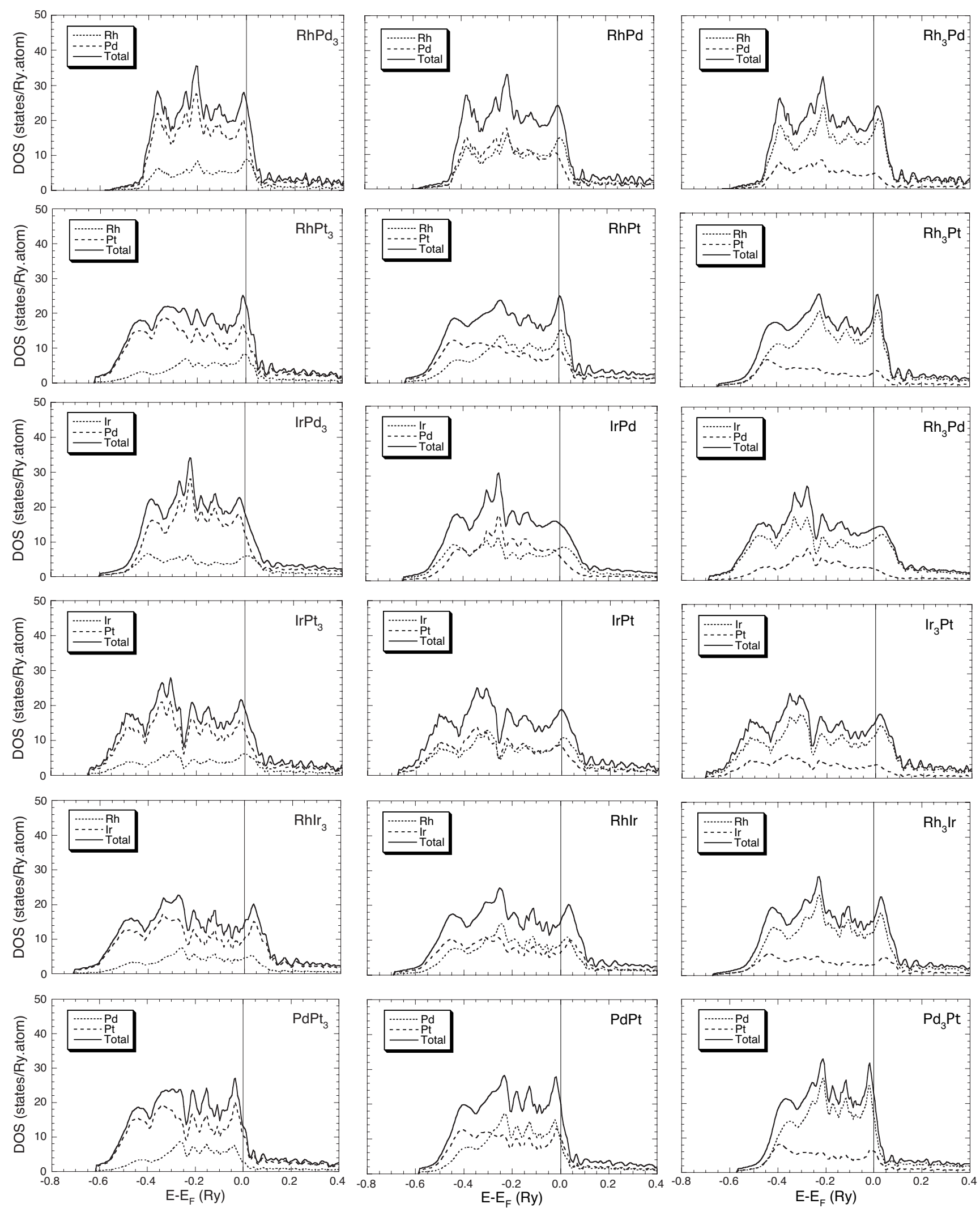

FIG. 3. Turchi et al. 


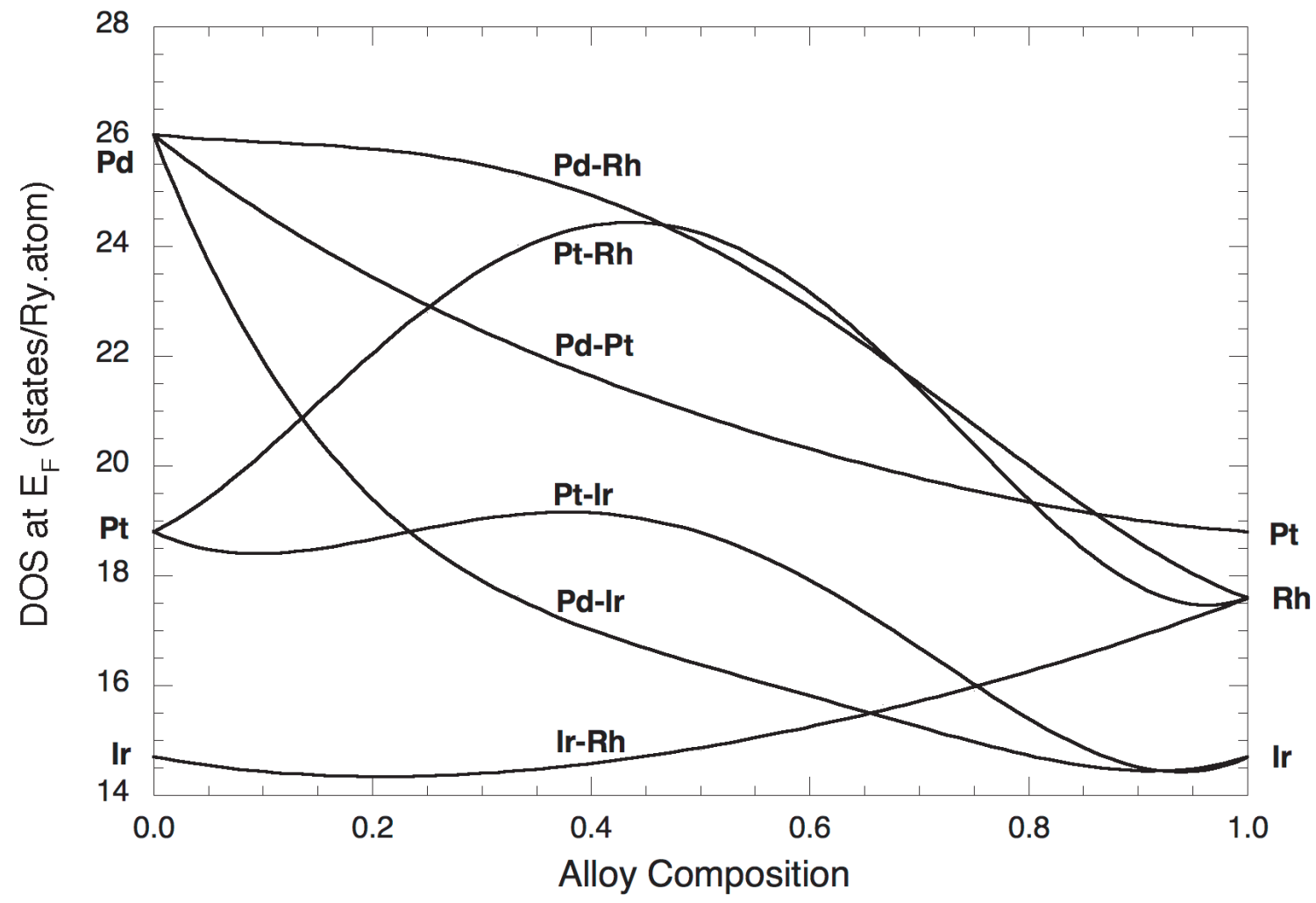

FIG. 4. Turchi et al. 


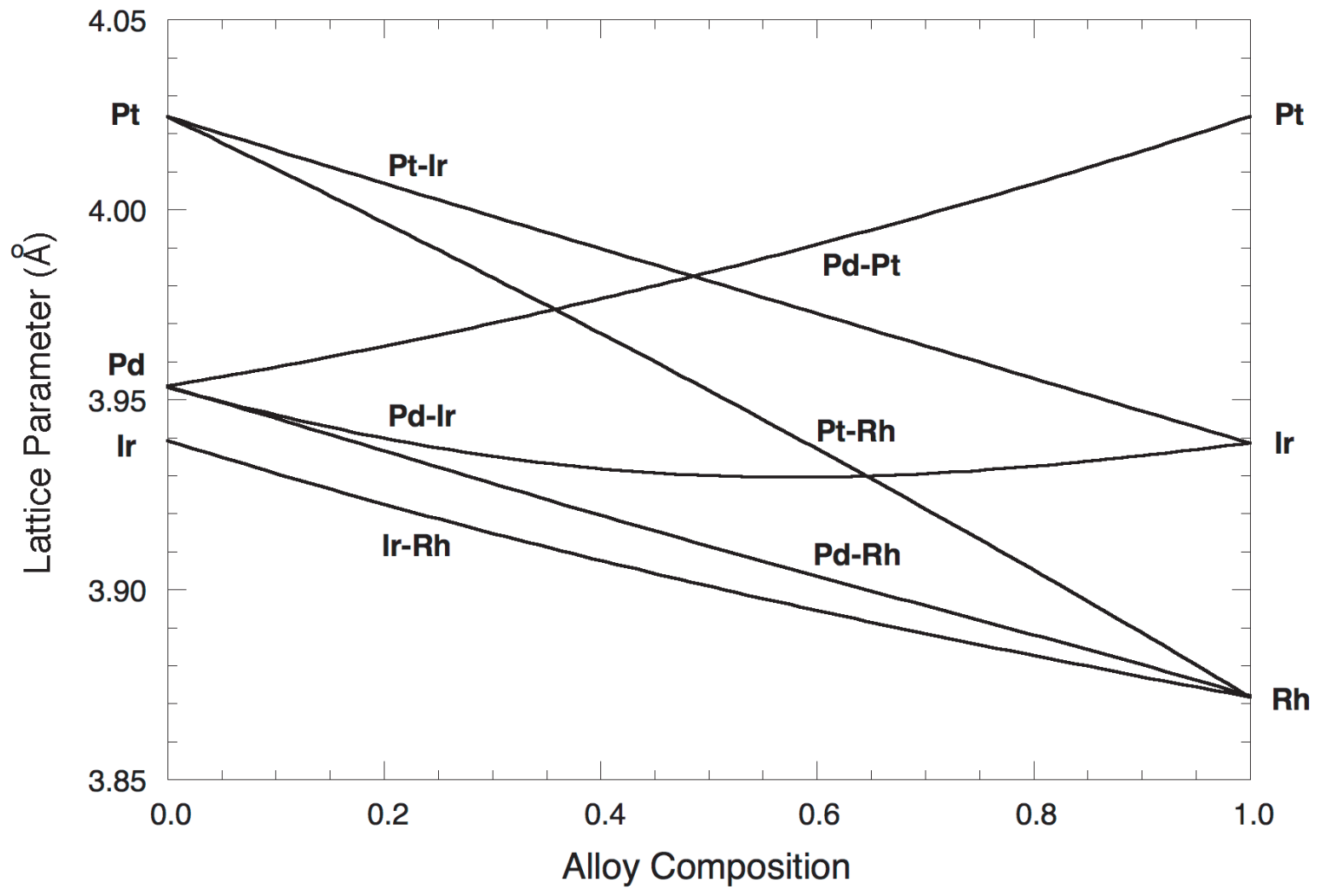

FIG. 5. Turchi et al. 


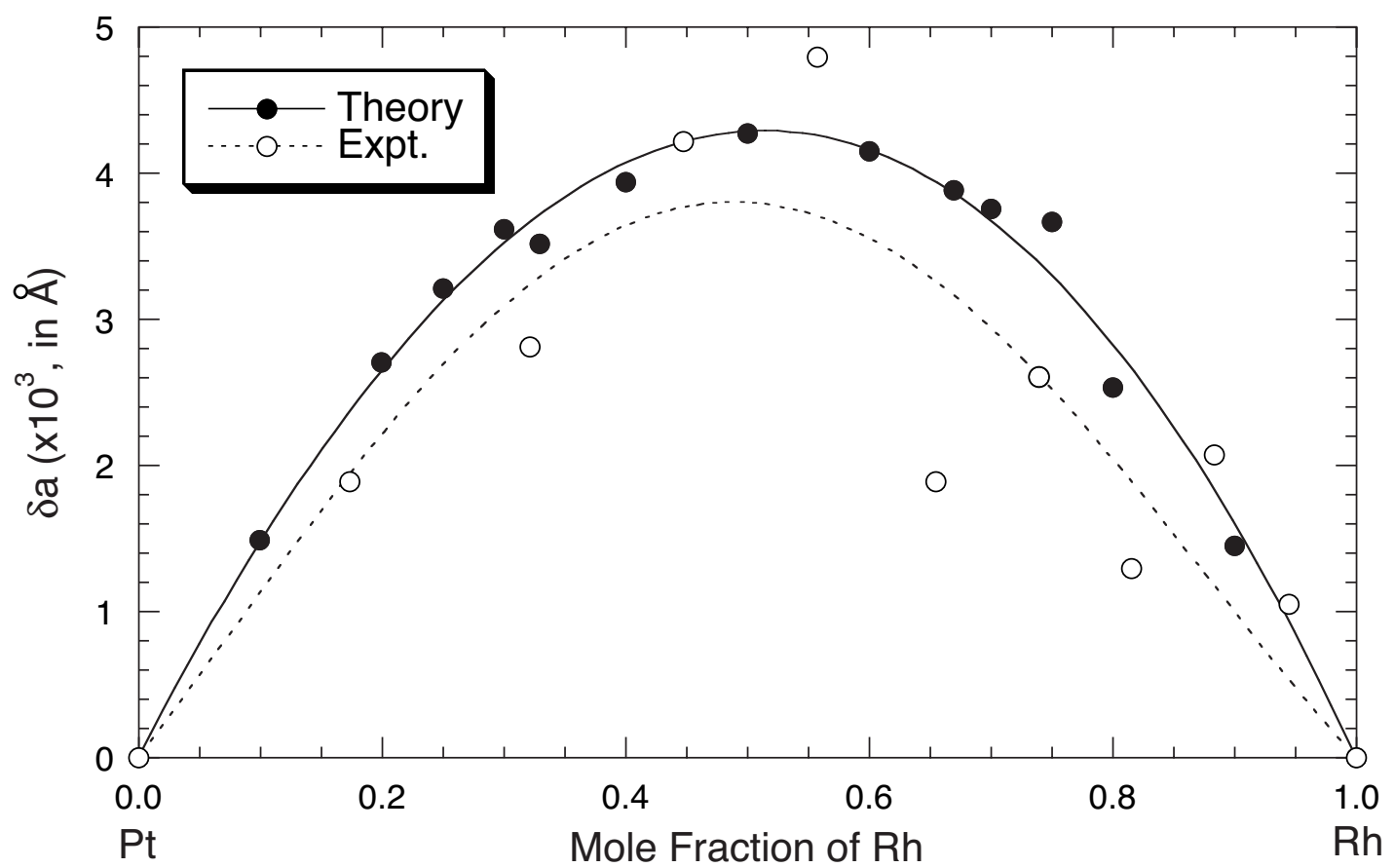

FIG. 6. Turchi et al. 


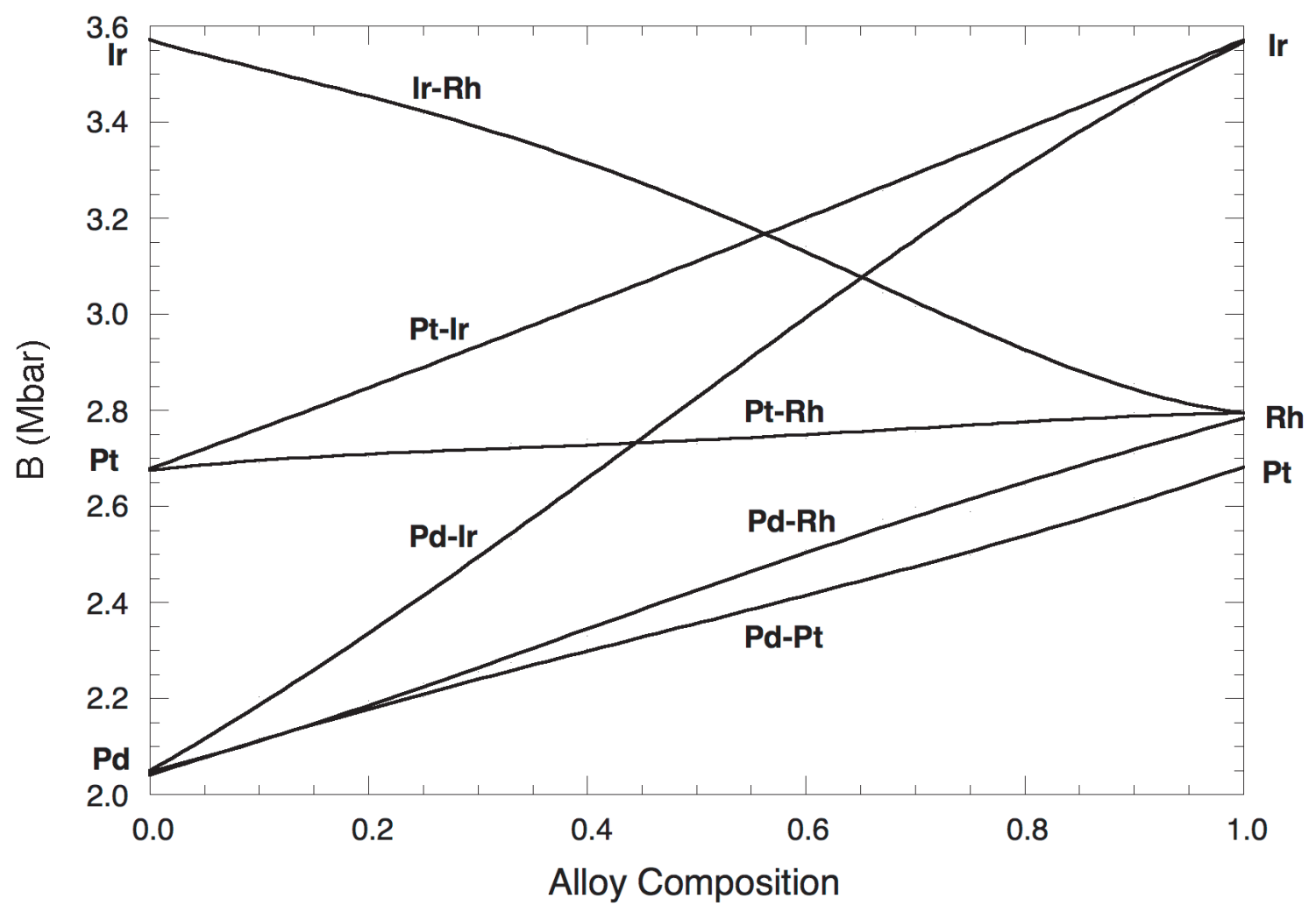

FIG. 7. Turchi et al. 

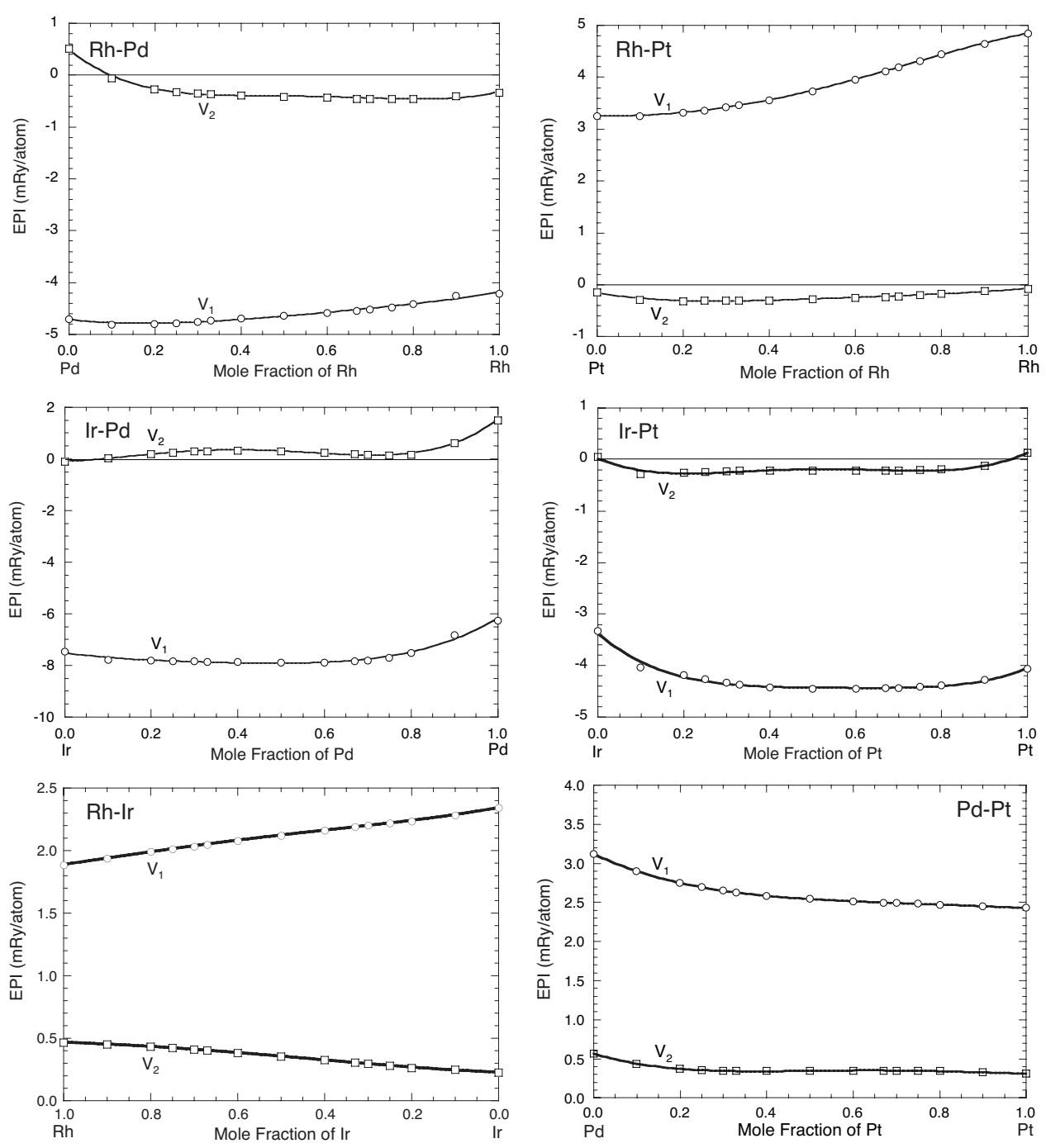

FIG. 8. Turchi et al. 


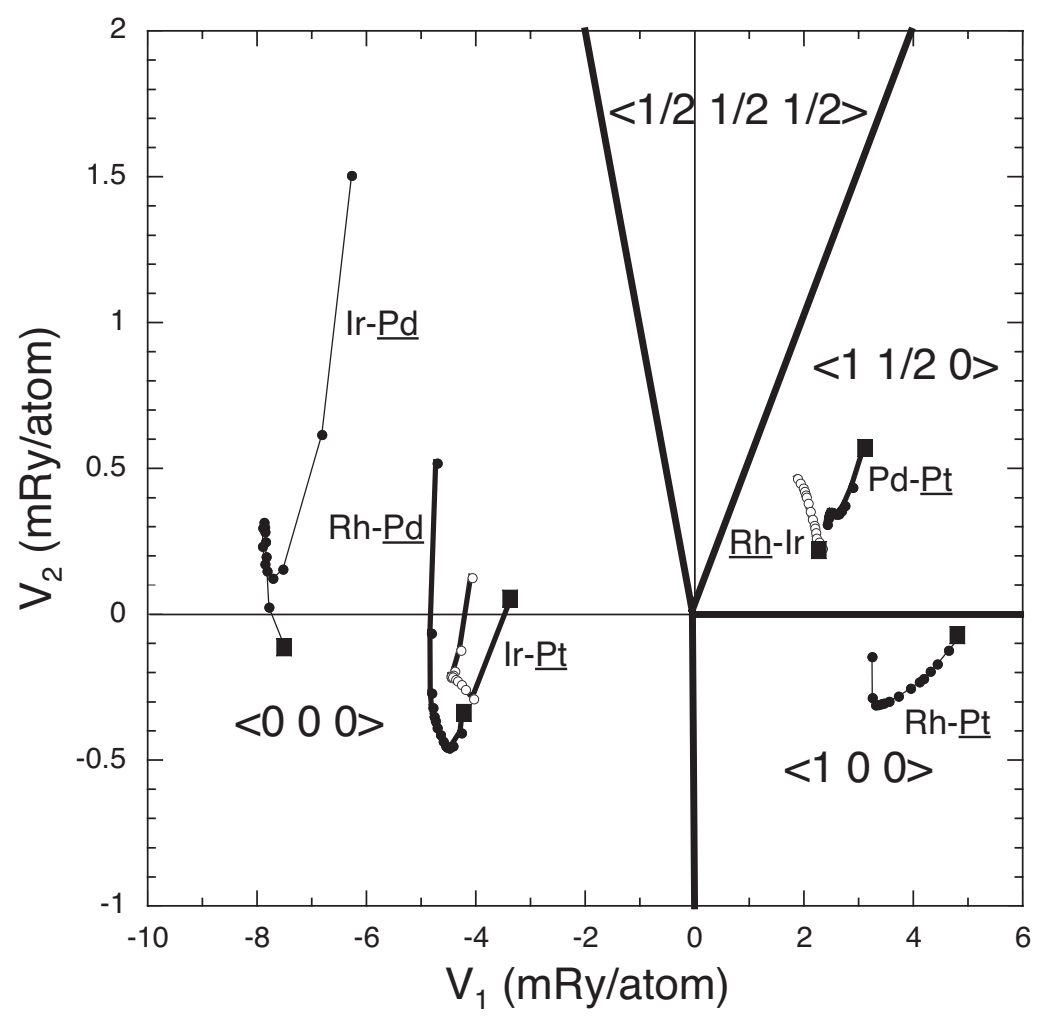

FIG. 9. Turchi et al. 

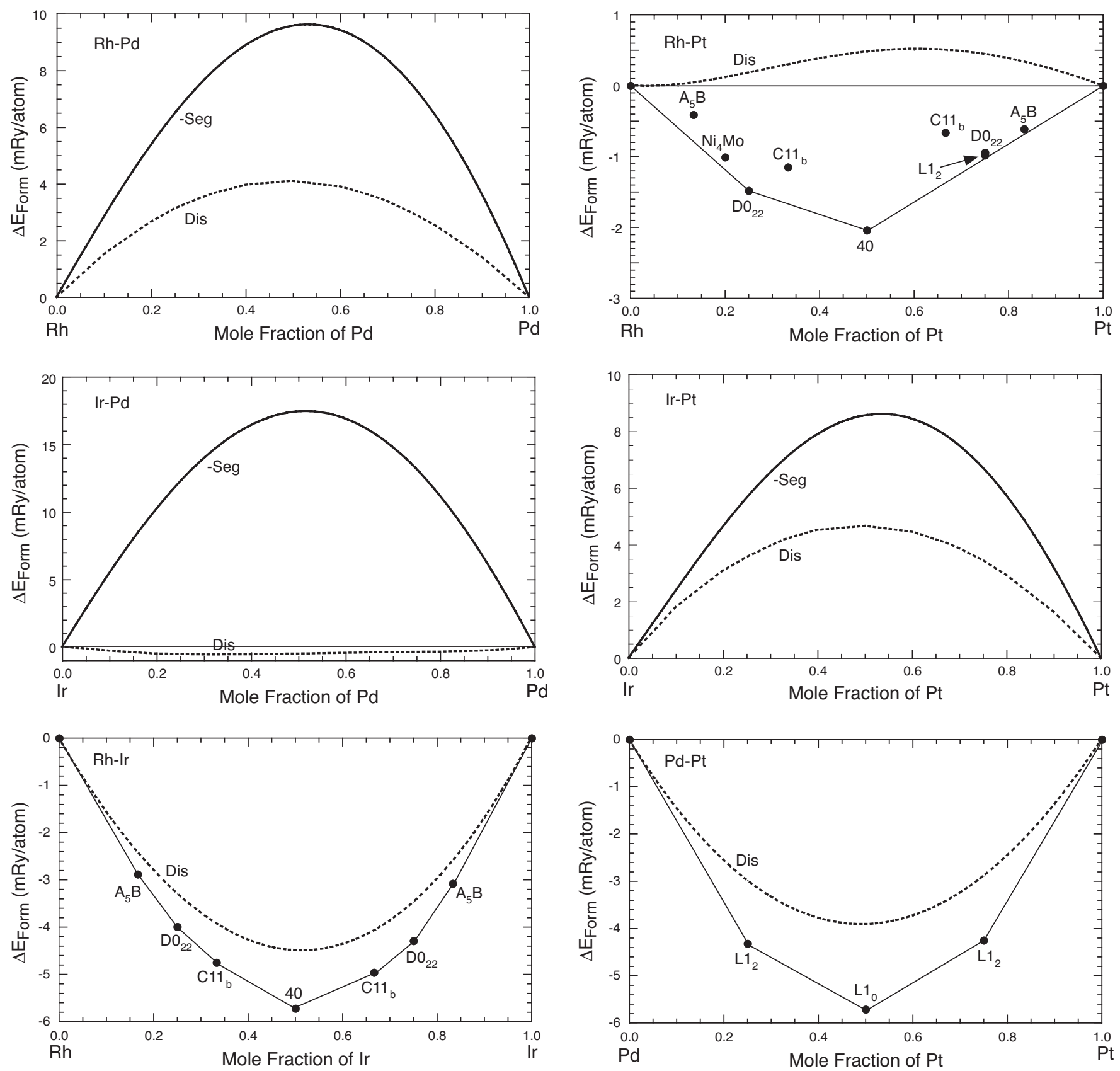

FIG. 10. Turchi et al. 


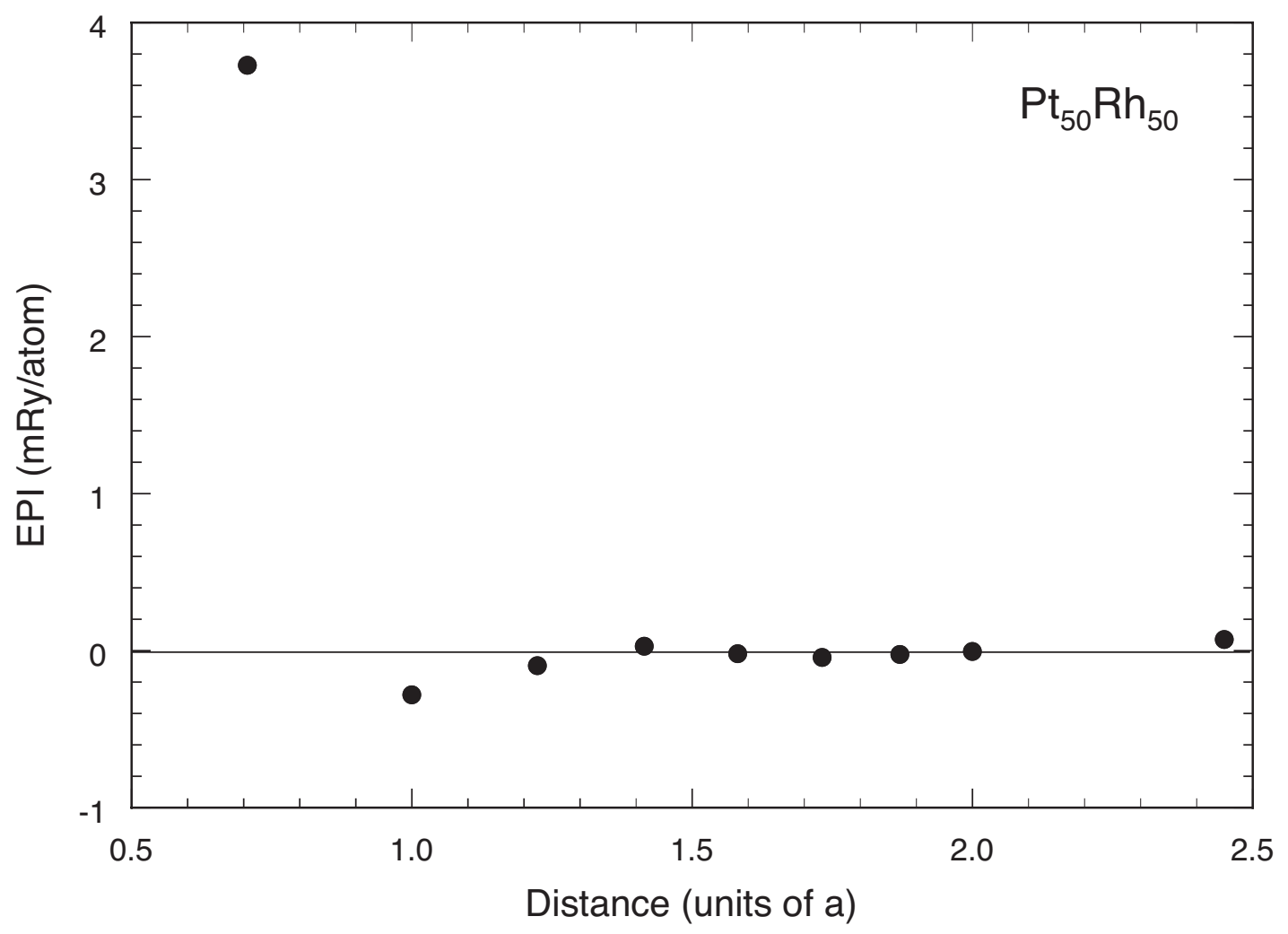

FIG. 11. Turchi et al. 